\title{
Leucine-rich Repeat Kinase 2 (LRRK2) Phosphorylates Rab10 in Glia and Neurons
}

\author{
Dong Hwan Ho ${ }^{1, \$, *}$, Daleum Nam, 1,,*, Mi Kyoung Seo ${ }^{2, *}$, Sung Woo Park ${ }^{2,3, * *}$, \\ Ilhong Son ${ }^{1,4, ;, * *}$ and Wongi Seol ${ }^{1, \dagger, * *}$ \\ ${ }^{1}$ InAm Neuroscience Research Center, Sanbon Medical Center, College of Medicine, \\ Wonkwang University, Gyeonggido 15865, Korea \\ ${ }^{2}$ Paik Institute for Clinical Research, Inje University College of Medicine, Busan 47392, Korea \\ ${ }^{3}$ Department of Convergence Biomedical Science, Inje University College of Medicine, Busan 47392, Korea \\ ${ }^{4}$ Department of Neurology, Sanbon Medical Center, College of Medicine, \\ Wonkwang University, Gyeonggido 15865, Korea
}

\begin{abstract}
Mutations in leucine-rich repeat kinase 2 (LRRK2) are the most common genetic cause of Parkinson's disease (PD). LRRK2 contains a functional kinase and GTPase domains. A pathogenic G2019S mutation that is the most prevalent among the LRRK2 mutations and is also found in sporadic cases, increases its kinase activity. Therefore, identification of LRRK2 kinase substrates and the development of kinase inhibitors are under intensive investigation to find PD therapeutics. Several recent studies have suggested members of Rab proteins, a branch of the GTPase superfamily, as LRRK2 kinase substrates. Rab proteins are key regulators of cellular vesicle trafficking. Among more than 60 members of human Rab proteins, Rab3, Rab5, Rab8, Rab10, Rab12, Rab29, Rab35, and Rab43 have been identified as LRRK2 kinase substrates. However, most studies have used human embryonic kidney (HEK) 293T cells overexpressing LRRK2/Rab proteins or murine embryonic fibroblast (MEF) cells which are not relevant to PD, rather than neuronal cells. In this study, we tested whether Rab proteins are phosphorylated by LRRK2 in astroglia in addition to neurons. Among the various Rab substrates, we tested phosphorylation of Rab10, because of the commercial availability and credibility of the phospho-Rab10 (pRab10) antibody, in combination with a specific LRRK2 kinase inhibitor. Based on the results of specific LRRK2 kinase inhibitor treatment, we concluded that LRRK2 phosphorylates Rab10 in the tested brain cells such as primary neurons, astrocytes and BV2 microglial cells.
\end{abstract}

Key Words: Astroglia, Kinase, LRRK2, Parkinson's Disease, Rab10

Received: April 19, 2019 / Revised: June 10, 2019 / Accepted: June 13, 2019

* Researcher, ${ }^{* *}$ Professor.

$\S_{\text {These authors contributed equally. }}$

${ }^{\dagger}$ Corresponding author: Ilhong Son. InAm Neuroscience Research Center, Sanbon Medical Center, College of Medicine, Wonkwang University, Sanbonro 321, Gunposi, Gyeonggido 15865, Korea.

Tel: +82-31-390-2486, Fax: +82-31-390-2414, e-mail: sonih@wku.ac.kr

† Corresponding author: Wongi Seol. InAm Neuroscience Research Center, Sanbon Medical Center, College of Medicine, Wonkwang University, Sanbonro 321, Gunposi, Gyeonggido 15865, Korea.

Tel: +82-31-390-2411, Fax: +82-31-390-2414, e-mail: wseolha@gmail.com

(C) The Korean Society for Biomedical Laboratory Sciences. All rights reserved.

(c) This is an Open Access article distributed under the terms of the Creative Commons Attribution Non-Commercial License (http://creativecommons.org/licenses/by-nc/3.0/) which permits unrestricted non-commercial use, distribution, and reproduction in any medium, provided the original work is properly cited. 


\section{INTRODUCTION}

Parkinson's disease (PD) is the second most common neurodegenerative disorder and can be inherited through genetic mutations (Lubbe and Morris, 2014). Among several known PD causing genes such as LRRK2, PARK2 (parkin), PINK1 and SNCA, LRRK2 is an autosomal dominant type of the familial PD gene (Belin and Westerlund, 2008) representing the most common genetic cause. LRRK2 contains functional GTPase and kinase domains (Monfrini and Di Fonzo, 2017). The pathogenic G2019S and R1441C mutations of LRRK2 are present in these enzyme domains and they increase its kinase activity (Seol, 2010; Steger et al., 2016).

Identifying the cellular substrates of LRRK2 kinase has been one of the goals in intense LRRK2 research and various studies have provided candidates such as moesin, tau, Rab5, p53, snapin and endophilinA (Parisiadou et al., 2009; Kawakami et al., 2012; Matta et al., 2012; Yun et al., 2013; Ho et al., 2015; Yun et al., 2015). Among the members of $\mathrm{Rab}$ proteins that regulate vesicle trafficking, recent studies have identified Rab 3A/B/C/D, Rab8A/B, Rab10, Rab12, Rab35, and Rab43, as endogenous substrates of LRRK2 kinase, along with Rab5B/C and Rab29 as putative substrates (Steger et al., 2017; Fujimoto et al., 2018). Recent studies (Steger et al., 2016; Steger et al., 2017; Eguchi et al., 2018; Fujimoto et al., 2018; Purlyte et al., 2018) have confirmed that LRRK2 is a critical regulator of cellular vesicle trafficking as suggested earlier by biochemical and cellular studies (Shin et al., 2008; Piccoli et al., 2011; Matta et al., 2012; Piccoli et al., 2014; Arranz et al., 2015; Soukup et al., 2016). However, most of these studies used MEF or HEK $293 \mathrm{~T}$ cells, which are not relevant to PD. Recent studies have reported that phosphorylation of Rab10 in human peripheral mononuclear blood cells (PBMCs) and human dopaminergic neuronal SH-SY5Y cells is inhibited by treatment with LRRK2 kinase inhibitors (Thirstrup et al., 2017; Liu et al., 2018) and the phosphorylation of Rab10 is activated in substantia nigra dopamine neurons in the postmortem brain tissue of idiopathic PD patients (Di Maio et al., 2018). However, no study reported the phosphorylation of Rab proteins by LRRK2 in brain cells other than neurons despite microglia and astrocytes accounting for the majority of brain cells and playing various critical roles in PD progression (Joe et al., 2018). LRRK2 is expressed in glial cells in addition to neurons, and LRRK2 activation and its regulatory roles in neuroinflammation have been reported in lipopolysaccharide (LPS)-activated microglia (Kim et al., 2012; Moehle et al., 2012; Ho et al., 2018). In addition, the effect of neuroinflammation on PD has been well established (Hirsch et al., 2012; Russo et al., 2015). Therefore, determining whether LRRK2 regulates immune functions via phosphorylation of Rab proteins in glial cells in addition to neurons is critical. Hence, we aimed to resolve whether LRRK2 can phosphorylate Rab proteins in these brain immune cells. In this study, we tested only the status of Rab10 phosphorylation because only the phospho-Rab10 (pRab10) antibody is credible as being widely used and has demonstrated species specificity among commercial phospho-Rab antibodies (Fan et al., 2018; Lis et al., 2018). We compared levels of Rab10 phosphorylation before and after LRRK2 kinase inhibitor treatment in murine BV2 microglia and rat primary astrocytes in addition to neurons, and analyzed them.

\section{MATERIALS AND METHODS}

\section{Materials and antibodies}

GSK2578215A (GSK, Tocris Bioscience, Bristol, UK, 4629), Lipopolysaccharide (LPS, Sigma, St. Louis, MO, USA), Pam3CSK4 (TLR2 ligand, InvivoGen, San Diego, CA, USA, tlrl-pms) and rotenone (Sigma, R8875) were purchased. The following antibodies were used for this study: LRRK2 (N241A/34, NeuroMabs, Davis, Ca, USA; 75-253, 1:1,000), pS935-LRRK2 (UDD210(12), Abcam, Cambridge, UK, ab133450, 1:5,000), $\alpha$-tubulin (DM1A, Sigma, T9026, 1:10,000), Rab10 (Abcam, ab104859, 1:1,000), pT73-Rab10 (Abcam, ab230261, 1:1,000), and horseradish peroxidaseconjugated goat anti-rabbit or anti-mouse IgG (Jackson Immuno Research. West Grove, PA, USA; 111-035-003 or 115-035-003, both 1:5,000).

\section{Cell culture and western blot analysis}

Rat primary astroglial cells were prepared from P1 stages 
of neonatal rats and attached to a flask, and microglia were isolated as previously described (Ho et al., 2018). Rat primary astrocytes were detached from the same flask surface using TrypLE Express (Gibco, Carlsbad, CA, USA, 12604013) and frozen as a stock. Dulbecco's modified Eagle's medium// F-12 (Gibco) with $10 \%$ fetal bovine serum and $1 \%$ penicillinstreptomycin was used as a medium to thaw and grow astrocytes. On day 5 after thawing, the primary astrocytes were treated with the indicated chemical reagents for the indicated times. Rat primary neuronal cells were isolated and cultured as previously described (Jang et al., 2018). Murine BV2 microglial cells were also cultured as previously described (Ho et al., 2018).

Animal experiments proceeded with the approval from the Committee for Animal Experimentation and the Institutional Animal Laboratory Review Board of Inje Medical
College (approval No. 2016-044).

To activate LRRK2 expression, cultures of astrocytes, micro glia and neurons were separately treated with the activators, Pam3CSK4 (400 ng/mL), LPS ( $1 \mu \mathrm{g} / \mathrm{mL})$, and rotenone $(20 \mu \mathrm{M})$ for $24 \mathrm{~h}$, respectively. When indicated, an LRRK2 specific kinase inhibitor, GSK $(3 \mu \mathrm{M})$, was added to each culture for $24 \mathrm{~h}$.

Cells were harvested and lysed with 1 x sodium dodecyl sulfate (SDS) sample loading buffer. The lysates were loaded for SDS-polyacrylamide gel electrophoresis (SDS-PAGE) without a centrifugation step and each protein level was analyzed using the indicated antibody for western blotting.

\section{Statistical analysis}

Western blotting data were analyzed as previously described (Ho et al., 2018). In brief, specific bands of the blots
(A)
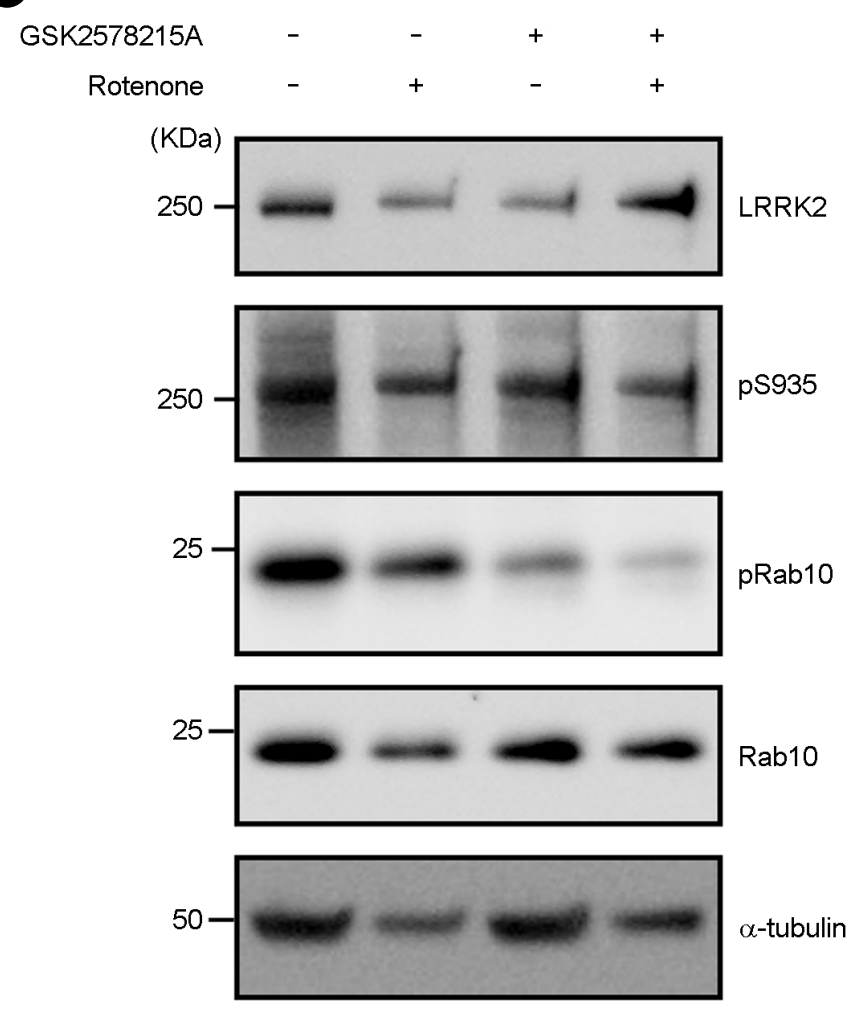

B

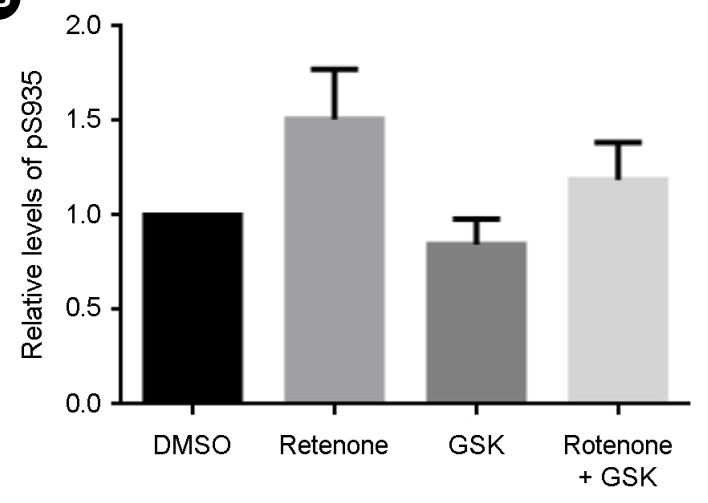

C

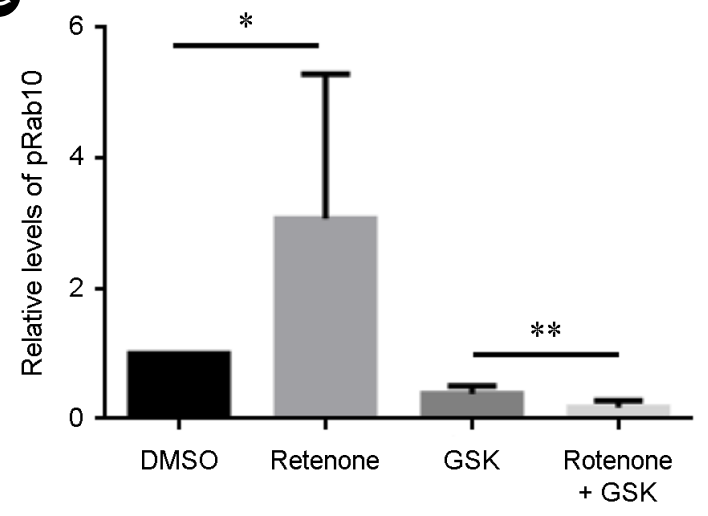

Fig. 1. Phospho-Rab10 level was reduced by LRRK2 kinase inhibitor treatment in rat primary neurons. The neurons were treated with or without rotenone $(20 \mu \mathrm{M})$ and GSK2578215A $(3 \mu \mathrm{M})$ for $24 \mathrm{~h}, 10$ days after seeding. The cell lysates were subjected to SDS-PAGE and analyzed with the indicated antibodies. A. A representative of western blot analysis after the indicated treatment. $(n=6) B$. C. Relative densitometric levels of pS935-LRRK2 which were normalized to $\alpha$-tubulin (B) and pRab10/Rab10 (C). $* P<0.05$, $* * P<0.01$. 
were imaged using Prism 6.0 (GraphPad, San Diego, CA, USA), and data were expressed as the mean \pm standard error of the mean (SEM). The statistical analysis was performed using a one-way analysis of variance (ANOVA) with Tukey's post-hoc test.

\section{RESULTS}

\section{LRRK2 phosphorylates endogenous Rab10 in primary neurons}

First, we investigated phosphorylated Rab10 levels in rat primary neurons to verify the findings of a previous study reporting that the pRab10 level was increased in substantia nigra dopamine neurons in the postmortem brain tissue of idiopathic PD patients (Di Maio et al., 2018). We treated rat primary neurons with or without LRRK2 kinase inhibitor, GSK, and compared the pRab10 level of each sample.
Rotenone was also used as a treatment because it has been reported to induce LRRK2 kinase activity (Jang et al., 2018). As expected, rotenone was cytotoxic and yielded reduced numbers of cells, resulting in a lower loading amount when the same volume of the cell lysate was used for SDSPAGE. The results showed that, when normalized, rotenone and rotenone+GSK treatments activate and reduce LRRK2 kinase activity, respectively, based on the pS935 LRRK2 levels; however, the differences were not significant (Fig. 1A, B). Similarly, the pRab10 levels were significantly increased by rotenone treatment, but reduced by GSK treatment (Fig. 1A, C).

\section{LRRK2 phosphorylates endogenous Rab10 in BV2 microglial cells}

Next, we tested murine BV2 microglial cells because they are more easily cultured than primary microglia. We
A

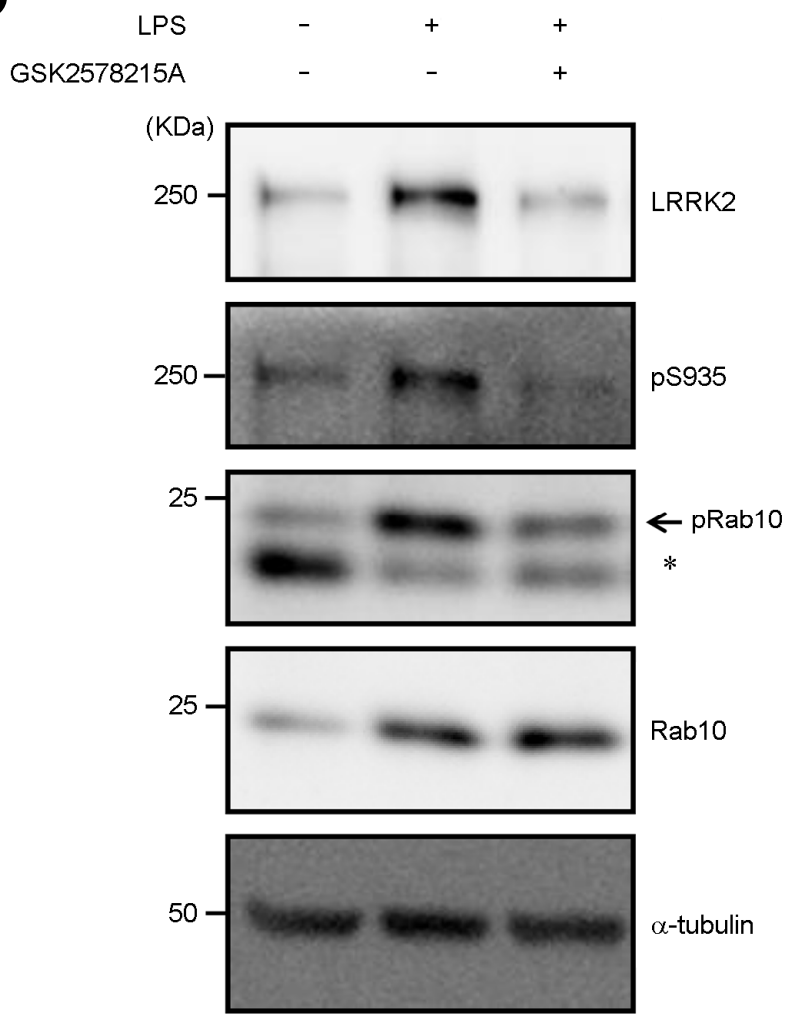

B

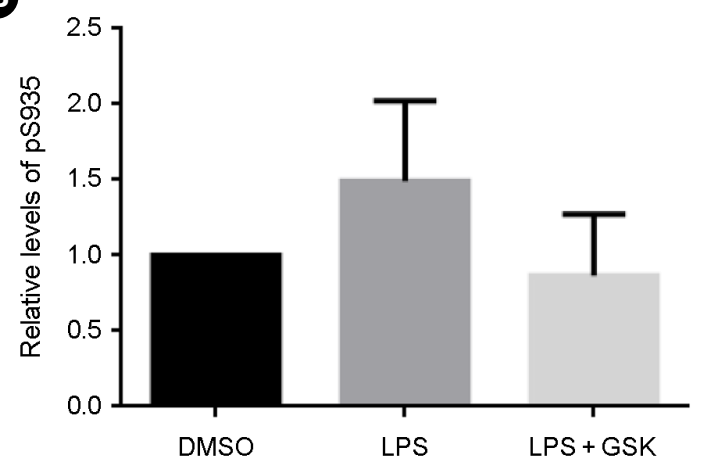

C

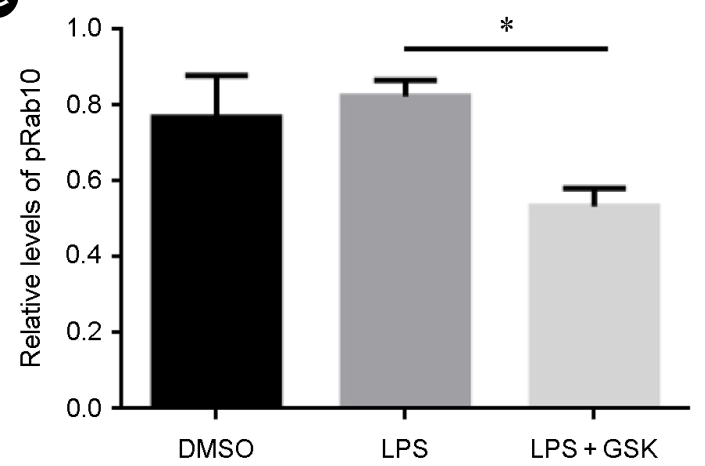

Fig. 2. Phospho-Rab10 level was reduced by LRRK2 kinase inhibitor treatment in murine BV2 microglial cells. Five days after seeding, the cells were treated with or without Pam3CSK4 (P3C4, $400 \mathrm{ng} / \mathrm{mL}$ ), a TLR2 ligand, and GSK2578215A (3 $\mu \mathrm{M})$ for $24 \mathrm{~h}$. A. A representative of western blot analysis after the indicated treatment. (*nonspecific band; $n=3$ ) B. C. Relative densitometric levels of pS935-LRRK2 which were normalized to $\alpha$-tubulin (B) and pRab10/Rab10 (C). $* P<0.05$. 
(A)
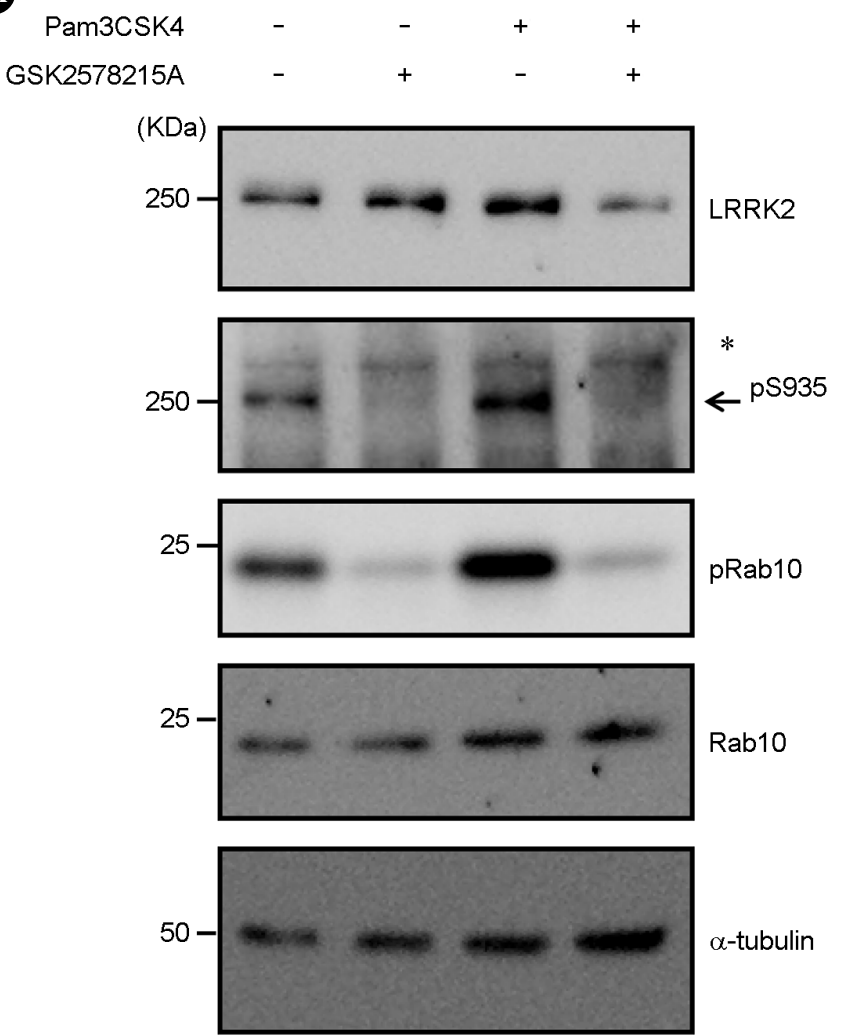

B

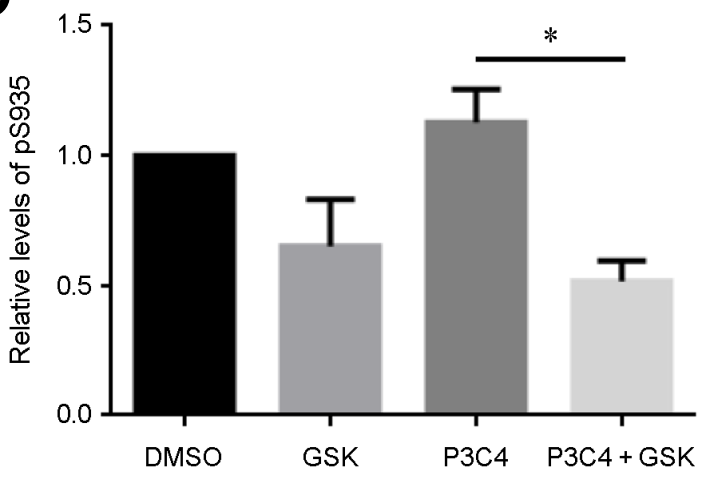

C

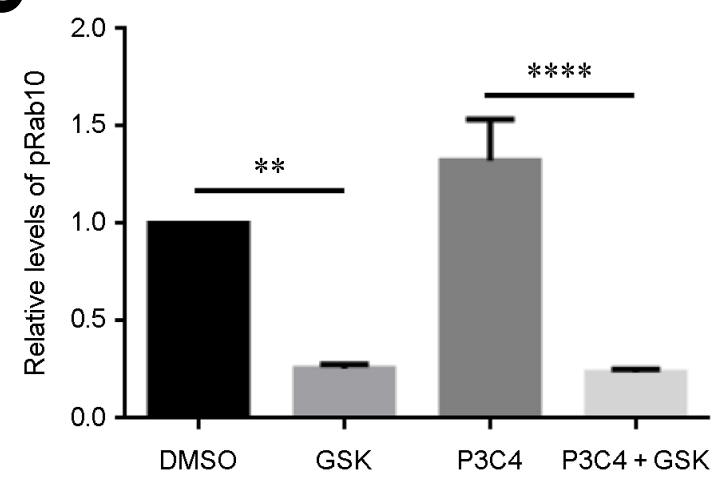

Fig. 3. The Phospho-Rab10 level was reduced by LRRK2 kinase inhibitor treatment in rat primary astrocytes. The cells were treated with or without lipopolysaccharide (LPS, $1 \mu \mathrm{g} / \mathrm{mL}$ ) and GSK2578215A $(3 \mu \mathrm{M})$ for $24 \mathrm{~h}$. The cell lysates were subjected to SDS-PAGE and analyzed with the indicated antibodies. A. A representative of western blot analysis after indicated treatment. (*nonspecific band; $n=3)$ B. C. Relative densitometric levels of pS935-LRRK2 which were normalized to $\alpha$-tubulin (B) and pRab10/Rab10 (C). *P<0.05, **P<0.01, $* * * * P<0.0001$.

treated them with LPS, a well-known activator of LRRK2 in microglia (Kim et al., 2012; Moehle et al., 2012; Ho et al., 2018). The result was similar to the pattern observed in the neuron cells. LPS treatments activated LRRK2 kinase activity, but LPS+GSK reduced the activity based on their pS935 LRRK2 levels; however, the differences were not significant (Fig. 2A, B). The small increase of the pRab10 levels induced by LPS treatment was significantly reduced by LPS+GSK treatment (Fig. 2A, C). Primary microglia were also tested, but only the pRab10, Rab10 and $\alpha$-tubulin signals could be detected, not the LRRK2 or pS935 level. This may have been because the number of cells used was too small. A primary microglia culture isolated from the neonatal rat brain typically results in a low yield (Jose et al., 2015). However, a pattern of pRab10 normalized by $\alpha$ tubulin in primary microglia was similar to that of BV2: significant increases and reductions induced by LPS and LPS+GSK treatments, respectively (data not shown). This also suggested that LRRK2 kinase phosphorylates endogenous Rab10 in microglia.

\section{LRRK2 phosphorylates endogenous Rab10 in primary astrocytes}

Primary astrocytes were treated with Pam3CSK4, a TLR2 ligand that activates astrocytes (Gurley et al., 2008), and GSK. Subsequently, their pRab10 and pS935 levels were analyzed. The results were similar to the pattern observed in the other cell types. Treatment with Pam3CSK4 and Pam3CSK4+ GSK significantly activated and reduced the pS935 levels, respectively (Fig. 3A, B). As expected, GSK treatment significantly reduced the pRab10 levels (Fig. 3A, C).

Overall, our data suggested that, for the first time that 
Rab10, at least, is phosphorylated by LRRK2 kinase activity in brain immune cells and neurons that are relevant to PD.

\section{DISCUSSION}

In this study, we tested only the phosphorylation of Rab10. At the time this study began, several phospho-Rab antibodies (e.g., Rab8, Rab10, and Rab29) had been reported and commercialized. However, only the phospho-Thr 73 Rab10 antibody specifically detects pRab10 in every tested cells, but phospho-Rab8 detects phospho-Rab8 as well as the phosphorylated forms of Rab10 and Rab35 (Lis et al., 2018). We also attempted to use the phospho-Rab29 antibody (MRC, Dundee, SA137) (Purlyte et al., 2018), but failed to detect a specific signal of endogenous protein in western blotting of SH-SY5Y cells. Therefore, we used only the phospho-Thr 73 Rab10 antibody. Hence, the results should be interpreted with caution and limited to only Rab10, rather than other Rab proteins that are LRRK2 kinase substrates. A recent study reported that LRRK2 genetic variants increase pRab10 levels, but not phospho-Rab12 in HEK293 cells (Christensen et al., 2018), suggesting that LRRK2 kinase activity may differentially regulate the phosphorylation of other Rab members.

Our result demonstrated that activation of Rab10 phosphorylation by LRRK2 kinase in brain immune cells as well as neurons. Similarly, several studies have reported that LRRK2 phosphorylates the Rab10 protein in immunestimulated human PBMCs (Thirstrup et al., 2017) although exact identities of such immune cells remain a subject to debate (Fan et al., 2018).

Rab10 phosphorylation may be mediated by other kinases in addition to LRRK2 kinase. This may be highly possible in cells and tissues in which the expression of Rab10 is higher than that of LRRK2. Notably, a considerable level of pRab10 remained after GSK treatment in BV2 cells (Fig. 2) whereas it was almost entirely absent in primary astrocytes and neurons (Fig. 1 and 3), suggesting that another functional kinase phosphorylates Rab10 in microglia.

Our previous study showed that GSK treatment of BV2 cells and rat primary microglia cells alleviates inflammation responses such as tumor necrosis factor alpha (TNF $\alpha)$ secre- tion which was increased by LPS treatment (Ho et al., 2018). A recent study suggested that phosphorylation of Rab10 and interleukin- 8 levels in neutrophil and peripheral blood mononuclear cells whose properties are somewhat similar to brain immune cells, have positive correlations although it did not investigate effect of LRRK2 kinase inhibitor (Atashrazm et al., 2019).

In addition, we aimed to determine whether the phosphorylation of Rab10 changed secretion of brain derived neurotrophic factor (BDNF) in astrocyte by western blot analysis because activated astrocytes increase the secretion of BDNF (Koyama et al., 2003). However, we were unable to detect their signals (data not shown), possibly because the activation of the corresponding cell types were too low. Therefore, the functional changes resulting from LRRK2mediated Rab10 phosphoryaltion warrant further investigation, although it seems to be related to activation of cytokine secretion.

Collectively, the results suggest that LRRK2 kinase activity mediates neuroinflammation in astroglia via Rab10 phosphorylation.

\section{ACKNOWLEDGEMENT}

This study was supported by the Basic Science Research Program (2018R1D1 A1B07041153) through the National Research Foundation (NRF) funded by the Ministry of Education, Science and Technology, Republic of Korea.

\section{CONFLICT OF INTEREST}

The authors declare no conflict of interest.

\section{REFERENCES}

Arranz AM, Delbroek L, Van Kolen K, Guimaraes MR, Mandemakers W, Daneels G, Matta S, Calafate S, Shaban H, Baatsen P, De Bock PJ, Gevaert K, Vanden Berghe P, Verstreken P, De Strooper B, Moechars D. LRRK2 functions in synaptic vesicle endocytosis through a kinase-dependent mechanism. J Cell Sci. 2015. 128: 541-552.

Atashrazm F, Hammond D, Perera G, Bolliger MF, Matar E, Halliday GM, Schule B, Lewis SJG, Nichols RJ, Dzamko N. LRRK2-mediated Rab10 phosphorylation in immune cells from Parkinson's disease patients. Mov Disord. 2019. 34: 406 
-415 .

Belin AC, Westerlund M. Parkinson's disease: a genetic perspective. Febs J. 2008. 275: 1377-1383.

Christensen KV, Hentzer M, Oppermann FS, Elschenbroich S, Dossang P, Thirstrup K, Egebjerg J, Williamson DS, Smith GP. LRRK2 exonic variants associated with Parkinson's disease augment phosphorylation levels for LRRK2-Ser1292 and Rab10-Thr73. BioRxiv. 2018. doi:?https://doi.org/10.1101/ 447946

Di Maio R, Hoffman EK, Rocha EM, Keeney MT, Sanders LH, De Miranda BR, Zharikov A, Van Laar A, Stepan AF, Lanz TA, Kofler JK, Burton EA, Alessi DR, Hastings TG, Greenamyre JT. LRRK2 activation in idiopathic Parkinson's disease. Sci Transl Med. 2018. 10: 451.

Eguchi T, Kuwahara T, Sakurai M, Komori T, Fujimoto T, Ito G, Yoshimura SI, Harada A, Fukuda M, Koike M, Iwatsubo T. LRRK2 and its substrate Rab GTPases are sequentially targeted onto stressed lysosomes and maintain their homeostasis. Proc Natl Acad Sci U S A. 2018. 115: E9115-E9124.

Fan Y, Howden AJM, Sarhan AR, Lis P, Ito G, Martinez TN, Brockmann K, Gasser T, Alessi DR, Sammler EM. Interrogating Parkinson's disease LRRK2 kinase pathway activity by assessing Rab10 phosphorylation in human neutrophils. Biochem J. 2018. 475: 23-44.

Fujimoto T, Kuwahara T, Eguchi T, Sakurai M, Komori T, Iwatsubo T. Parkinson's disease-associated mutant LRRK2 phosphorylates Rab7L1 and modifies trans-Golgi morphology. Biochem Biophys Res Commun. 2018. 495: 1708-1715.

Gurley C, Nichols J, Liu S, Phulwani NK, Esen N, Kielian T. Microglia and Astrocyte Activation by Toll-Like Receptor Ligands: Modulation by PPAR-gamma Agonists. PPAR Res. 2008. 2008: 453120.

Hirsch EC, Vyas S, Hunot S. Neuroinflammation in Parkinson's disease. Parkinsonism Relat Disord. 2012. 18 Suppl 1: S210 $-\mathrm{S} 212$.

Ho DH, Je AR, Lee H, Son I, Kweon HS, Kim HG, Seol W. LRRK2 Kinase Activity Induces Mitochondrial Fission in Microglia via Drp1 and Modulates Neuroinflammation. Exp Neurobiol. 2018. 27: 171-180.

Ho DH, Kim H, Kim J, Sim H, Ahn H, Kim J, Seo H, Chung KC, Park BJ, Son I, Seol W. Leucine-Rich Repeat Kinase 2 (LRRK2) phosphorylates p53 and induces p21 (WAF1/CIP1) expression. Mol Brain. 2015. 8: 54.

Jang J, Oh H, Nam D, Seol W, Seo MK, Park SW, Kim HG, Seo H, Son I, Ho DH, Increase in anti-apoptotic molecules, nucleolin, and heat shock protein 70, against upregulated LRRK2 kinase activity. Anim Cells Syst. (Seoul) 2018. 22: 273-280.

Joe EH, Choi DJ, An J, Eun JH, Jou I, Park S. Astrocytes, Microglia, and Parkinson's Disease. Exp Neurobiol. 2018. 27: 77-87.

Jose S, Tan SW, Tong CK, Vidyadaran S. Isolation and characterization of primary microglia from post-natal murine brain tissues: a comparison of two methods. Cell Biol Int. 2015. 39: 1355-1363.

Kawakami F, Yabata T, Ohta E, Maekawa T, Shimada N, Suzuki M, Maruyama H, Ichikawa T, Obata F. LRRK2 phosphorylates tubulin-associated tau but not the free molecule: LRRK2mediated regulation of the tau-tubulin association and neurite outgrowth. PLoS One. 2012. 7: e30834.

Kim B, Yang MS, Choi D, Kim JH, Kim HS, Seol W, Choi S, Jou I, Kim EY, Joe EH. Impaired inflammatory responses in murine Lrrk2-knockdown brain microglia. PLoS One. 2012. 7: e34693.

Koyama Y, Tsujikawa K, Matsuda T, Baba A. Intracerebroventricular administration of an endothelin ETB receptor agonist increases expressions of GDNF and BDNF in rat brain. Eur $\mathbf{J}$ Neurosci. 2003. 18: 887-894.

Lis P, Burel S, Steger M, Mann M, Brown F, Diez F, Tonelli F, Holton JL, Ho PW, Ho SL, Chou MY, Polinski NK, Martinez TN, Davies P, Alessi DR. Development of phospho-specific Rab protein antibodies to monitor in vivo activity of the LRRK2 Parkinson's disease kinase. Biochem J. 2018. 475: $1-22$.

Liu Z, Bryant N, Kumaran R, Beilina A, Abeliovich A, Cookson MR, West AB. LRRK2 phosphorylates membrane-bound Rabs and is activated by GTP-bound Rab7L1 to promote recruitment to the trans-Golgi network. Hum Mol Genet. 2018. 27: 385-395.

Lubbe S, Morris HR. Recent advances in Parkinson's disease genetics. J Neurol. 2014. 261: 259-266.

Matta S, Van Kolen K, da Cunha R, van den Bogaart G, Mandemakers W, Miskiewicz K, De Bock PJ, Morais VA, Vilain S, Haddad D, Delbroek L, Swerts J, Chavez-Gutierrez L, Esposito G, Daneels G, Karran E, Holt M, Gevaert K, Moechars DW, De Strooper BD, Verstreken P. LRRK2 Controls an EndoA Phosphorylation Cycle in Synaptic Endocytosis. Neuron. 2012. 75: 1008-1021.

Moehle MS, Webber PJ, Tse T, Sukar N, Standaert DG, DeSilva TM, Cowell RM, West AB. LRRK2 inhibition attenuates microglial inflammatory responses. J Neurosci. 2012. 32: 1602-1611.

Monfrini E, Di Fonzo A. Leucine-Rich Repeat Kinase (LRRK2) Genetics and Parkinson's Disease. Adv Neurobiol. 2017. 14: 
$3-30$.

Parisiadou L, Xie C, Cho HJ, Lin X, Gu XL, Long CX, Lobbestael

E, Baekelandt V, Taymans JM, Sun L, Cai H. Phosphorylation of ezrin/radixin/moesin proteins by LRRK2 promotes the rearrangement of actin cytoskeleton in neuronal morphogenesis. J Neurosci. 2009. 29: 13971-13980.

Piccoli G, Condliffe SB, Bauer M, Giesert F, Boldt K, De Astis S, Meixner A, Sarioglu H, Vogt-Weisenhorn DM, Wurst W, Gloeckner CJ, Matteoli M, Sala C, Ueffing M. LRRK2 Controls Synaptic Vesicle Storage and Mobilization within the Recycling Pool. J Neurosci. 2011. 31: 2225-2237.

Piccoli G, Onofri F, Cirnaru MD, Kaiser CJ, Jagtap P, Kastenmuller A, Pischedda F, Marte A, von Zweydorf F, Vogt A, Giesert F, Pan L, Antonucci F, Kiel C, Zhang M, Weinkauf S, Sattler M, Sala C, Matteoli M, Ueffing M, et al. Leucine-Rich Repeat Kinase 2 Binds to Neuronal Vesicles through Protein Interactions Mediated by Its C-Terminal WD40 Domain. Mol Cell Biol. 2014. 34: 2147-2161.

Purlyte E, Dhekne HS, Sarhan AR, Gomez R, Lis P, Wightman M, Martinez TN, Tonelli F, Pfeffer SR, Alessi DR. Rab29 activation of the Parkinson's disease-associated LRRK2 kinase. Embo J. 2018. 37: 1-18.

Russo I, Berti G, Plotegher N, Bernardo G, Filograna R, Bubacco

L, Greggio E. Leucine-rich repeat kinase 2 positively regulates inflammation and down-regulates NF-kappaB p50 signaling in cultured microglia cells. J Neuroinflammation. 2015. 12: 230 .

Seol W. Biochemical and molecular features of LRRK2 and its pathophysiological roles in Parkinson's disease. BMB Rep. 2010. 43: 233-244.

Shin N, Jeong H, Kwon J, Heo HY, Kwon JJ, Yun HJ, Kim CH, Han BS, Tong Y, Shen J, Hatano T, Hattori N, Kim K-S, Chang $\mathrm{S}$, Seol W. LRRK2 regulates synaptic vesicle endocytosis. Exp. Cell Res. 2008. 314: 2055-2065.
Soukup SF, Kuenen S, Vanhauwaert R, Manetsberger J, HernandezDiaz S, Swerts J, Schoovaerts N, Vilain S, Gounko NV, Vints K, Geens A, De Strooper B, Verstreken P. A LRRK2-Dependent EndophilinA Phosphoswitch Is Critical for Macroautophagy at Presynaptic Terminals. Neuron. 2016. 92: 829-844.

Steger M, Diez F, Dhekne HS, Lis P, Nirujogi RS, Karayel O, Tonelli F, Martinez TN, Lorentzen E, Pfeffer SR, Alessi DR, et al. Systematic proteomic analysis of LRRK2-mediated Rab GTPase phosphorylation establishes a connection to ciliogenesis. Elife. 2017. 6: e31012.

Steger M, Tonelli F, Ito G, Davies P, Trost M, Vetter M, Wachter S, Lorentzen E, Duddy G, Wilson S, Baptista MA, Fiske BK, Fell MJ, Morrow JA, Reith AD, Alessi DR, Mann. M. Phosphoproteomics reveals that Parkinson's disease kinase LRRK2 regulates a subset of Rab GTPases. Elife. 2016. 5: e12813.

Thirstrup K, Dachsel JC, Oppermann FS, Williamson DS, Smith GP, Fog K, Christensen KV. Selective LRRK2 kinase inhibition reduces phosphorylation of endogenous Rab10 and Rab12 in human peripheral mononuclear blood cells. Sci Rep. 2017. 7: 10300 .

Yun HJ, Kim H, Ga I, Oh H, Ho DH, Kim J, Seo H, Son I, Seol W. An early endosome regulator, Rab5b, is an LRRK2 kinase substrate. J Biochem. 2015. 157: 485-495.

Yun HJ, Park J, Ho DH, Kim H, Kim CH, Oh H, Ga I, Seo H, Chang S, Son I, Seol W. LRRK2 phosphorylates Snapin and inhibits interaction of Snapin with SNAP-25. Exp Mol Med. 2013. 45, e36.

https://doi.org/10.15616/BSL.2019.25.2.177

Cite this article as: Ho DH, Nam D, Seo MK, Park SW, Son I, Seol W. Leucine-rich Repeat Kinase 2 (LRRK2) Phosphorylates Rab10 in Glia and Neurons. Biomedical Science Letters. 2019. 25: 177-184. 